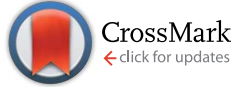

Cite this: Anal. Methods, 2016, 8, 1235

\title{
Detecting carbohydrate-lectin interactions using a fluorescent probe based on DBD dyes $\uparrow$
}

\author{
D. Bader, ${ }^{a}$ D. T. Klier, ${ }^{\text {a }}$ C. Hettrich, ${ }^{\text {b }}$ F. F. Bier ${ }^{\text {b }}$ and P. Wessig ${ }^{\star a}$
}

Received 14th November 2015

Accepted 13th January 2016

DOI: $10.1039 / c 5 a y 02991 \mathrm{k}$

www.rsc.org/methods

Herein we present an efficient synthesis of a biomimetic probe with modular construction that can be specifically bound by the mannose binding FimH protein - a surface adhesion protein of $E$. coli bacteria. The synthesis combines the new and interesting DBD dye with the carbohydrate ligand mannose via a Click reaction. We demonstrate the binding to $E$. coli bacteria over a large concentration range and also present some special characteristics of those molecules that are of particular interest for the application as a biosensor. In particular, the mix-and-measure ability and the very good photo-stability should be highlighted here.

Nowadays, fluorescent dyes play an extremely important role in biochemistry, medical diagnostics, and ingredient research. ${ }^{1}$ Despite the vast number of fluorophores hitherto used for these purposes, there is ongoing interest in new fluorophores. This is due to the diverse requirements of the applications which are not combined in any fluorescent dye. Excitation and absorbance wavelengths $\left(\lambda_{\text {abs }}, \lambda_{\text {exc }}\right)$, as well as the difference between them (Stokes shift, $\Delta \lambda$ ), extinction coefficient $(\varepsilon)$ and fluorescence quantum yield $\left(\Phi_{\mathrm{F}}\right)$, as well as their product (brightness, $\varepsilon \cdot \Phi_{\mathrm{F}}$ ), fluorescence lifetime $\left(\tau_{\mathrm{F}}\right)$, bleaching stability and environmental sensitivity are important parameters influencing the usability of a certain dye. Furthermore, the synthetic accessibility and the possibility to link the dye with various biomolecules are decisive criteria for successful application.

Recently, we have developed a new class of fluorescent dyes, whose structure is based on $[1,3]$ dioxolo[ $[4,5-f][1,3]$ benzo-dioxole (DBD, bold in Fig. 1) and we called them DBD dyes. ${ }^{2-7}$ These dyes are characterised by a large Stokes shift $(\Delta \lambda>100 \mathrm{~nm})$, combined with long fluorescence lifetimes $\left(\tau_{\mathrm{F}}>20 \mathrm{~ns}\right)$ and exceptional bleaching stability. $\tau_{\mathrm{F}}$ and $\Phi_{\mathrm{F}}$ of esters $\mathbf{1 b}$ are nearly independent of the polarity of the microenvironment, whereas

${ }^{a}$ Institut für Chemie, Universität Potsdam, Karl-Liebknecht-Str. 25-26, D-14476 Potsdam, Germany.E-mail: wessig@uni-potsdam.de

${ }^{b}$ Fraunhofer IZI-BB, Am Mühlenberg 13, D-14476 Potsdam, Germany

$\uparrow$ Electronic supplementary information (ESI) available: Synthetic procedures and compound characterisation. See DOI: 10.1039/c5ay02991k the respective parameters of ketones 1a exhibit a distinct sensitivity towards their environment. In aqueous surroundings the fluorescence of $\mathbf{1 a}$ is nearly completely quenched but it is "switched on" in a hydrophobic environment. ${ }^{2}$ These properties were already successfully exploited in probes for sensing lipophilic environments, such as micelles and biological membranes $^{3}$ and for detecting conformational changes of proteins. ${ }^{4,5}$ The environmental sensitivity was also used in the development of lifetime-based binding assays. ${ }^{6}$ Very recently, we have introduced a new FRET system with DBD dyes as an acceptor. $^{7}$

Inspired by these promising results we hypothesised that it should be possible to develop fluorescent probes by linking DBD fluorophores with small molecules, especially carbohydrates, and detect the specific binding event with proteins. Because the recognition of glycoproteins at the surface of human cells by membrane associated proteins of (pathogenic) bacteria is the key event of infection by these germs, this approach should offer the possibility to develop fluorescent probes for the detection of harmful pathogens.

Until now only few groups have been using carbohydrate fluorescent probes as biosensors. One approach is the usage of quantum dots (QDs) as a fluorescent marker. Because of their physical characteristics they have some important advantages like high bleaching stability. Robinson et al. and Coulon et al. introduced CdTe-QDs as water soluble biosensors by having
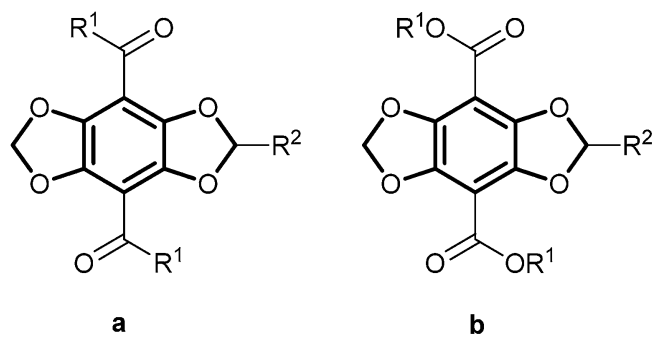

Fig. 1 Structure of DBD dyes of the first (1a) and second (1b) generation. 
carbohydrates adsorb at their surface. ${ }^{\mathbf{8 , 9}}$ However, the removal from the surface needs complicated washing procedures. A completely different method is the application of fluorescent glycolised BSA-proteins. ${ }^{\mathbf{1 0}}$ Kuchler et al. were able to stain endothelial cells. But the protein production is relatively complex and expensive and therefore not suitable for routine diagnostics. Another approach was introduced by Lee et al. in $2007 .{ }^{11}$ They described the formation of self-assembled glycolnanoribbons that were called triblock peptides. They consist of a mannose or glucose pyranoside, a PEG spacer and a $\beta$-sheet forming peptide. Nile red is encapsulated in their hydrophobic region and thus $E$. coli bacteria can be detected specifically and with high sensitivity. Nevertheless, this procedure bears the risk of hydrophobic interactions between the dye and target rather than nanoribbon mediated binding. This can cause false positive results. ${ }^{12}$

Herein we present a simple and modular synthesis of a monovalent carbohydrate fluorescent probe that was used as the biomimetic probe for the specific binding on lectins. To demonstrate the proof-of-principle of this approach we chose mannose as carbohydrate and the non-infectious $E$. coli bacteria strain DH5 $\alpha$. The DBD dye 2 of the first generation (1a, $c f$. Scheme 1), which was already successfully applied in protein binding assays, ${ }^{6}$ was used as the fluorescent marker. Based on the wide ranging dynamic fluorescence properties that depend on the polarity of the surrounding, the DBD dyes are particularly suitable for the application in the biosensor field. It was shown before that the DBD dye has an emission shift of $80 \mathrm{~nm}$ from nonpolar to polar solvents. Additionally, it has a Stokes shift up to $157 \mathrm{~nm}$ and a high dynamic in the quantum yields. In very polar surroundings, e.g. in water, it is approximately zero, but it increases up to $86 \%$ in nonpolar solvents. The DBD dye was synthesized in only 8 steps in good to very good yields. ${ }^{2,4}$

The manno pyranoside $\mathbf{5}$ was used as the carbohydrate ligand and was synthesized in 2 steps from $\alpha$-D-manno-pyranoside 4. To connect the DBD dye to the mannose, we used the commercially available linker 3 with an azide group. Due to its structure the linker increases the water solubility of the whole molecule. In this synthesis we followed the method for propargylation of Kusumoto et al. that forms only the $\alpha$-pyranoside product. ${ }^{13} \mathrm{TMS}-\mathrm{Cl}$ is a mediator to form the propargylic ether in the 1-position. This synthesis works in moderate yields of $41 \%$. In a second step the free hydroxyl groups were protected by acetyl groups in quantitative yields to give $\mathbf{5}$.

The modular combination of the individual building blocks was realised in an orthogonal way by first formation of carboxylic amide $\mathbf{7}$ followed by a copper catalysed Click-reaction ${ }^{\mathbf{1 4}}$ to give $\mathbf{8}$. Both reactions result in good to very good yields with $66 \%$ and $84 \%$. It should be noted that we used the CuI/DIPEA catalyst for the latter reaction. ${ }^{\mathbf{1 4 c}}$ The final deprotection gives the desired biomimetic probe 6 in quantitative yield (Scheme 1).

The binding studies of $\mathbf{6}$ were performed on a fluorescence microscope. E. coli bacteria DH5 $\alpha$ were cultivated in growthmedium, washed and resuspended in PBS buffer and then incubated for one hour with compound $\mathbf{6}$ in different concentrations. After incubation the bacteria were washed again and then fixed on glass slides.
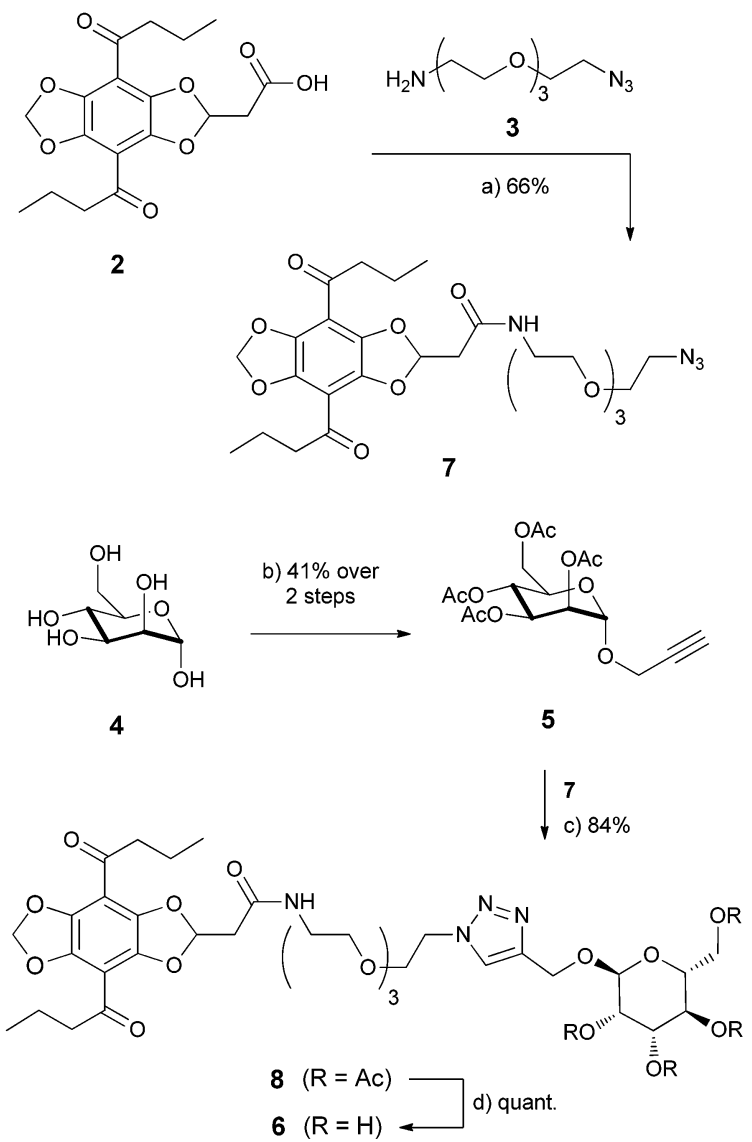

Scheme 1 Synthesis of compound 6 (a) 3.4 eq. N,N-diisopropylethylamine, 1.1 eq. PYBOP, 1.1 eq. $3, \mathrm{DCM}, 0{ }^{\circ} \mathrm{C}$-r.t., $5 \mathrm{~h}, 66 \%$. (b) (i) 10 eq. TMS-Cl, 25 eq. 2-propyn-1-ol, r.t., 5 d, 41\%; (ii) 10 eq. pyridin, 8 eq. $\mathrm{Ac}_{2} \mathrm{O}, 0{ }^{\circ} \mathrm{C}$-r.t., quant. (c) 1 eq. $\mathrm{N}, \mathrm{N}$-diisopropylethylamine (DIPEA), 1 eq. 5, 50 mol\% Cul, THF, r.t., 20 min, 84\%. (d) 5 eq. $\mathrm{NaOMe}, \mathrm{MeOH}, \mathrm{RT}$, 20 min, quant.

The images were made in different modes: bright field and fluorescence modes. The DBD derivative was excited at $\lambda_{\mathrm{ex}}=405$ $\mathrm{nm}$ and detected in an emission range from $\lambda_{\mathrm{em}}=530$ to 600 $\mathrm{nm}$. A concentration range of compound 6 from $10^{-3}$ to $10^{-12}$ mol L ${ }^{-1}$ was investigated.

In Fig. 2 we present only the highest (A) and the lowest (J) concentrations, as well as reference bacteria without treatment with $6(\mathrm{~W})$. A 2-channel image (1) where the bright field and fluorescence images were merged and then the according bright field (2) and fluorescence (3) images are displayed side by side. The bright field and 2-channel images were used as a first colocalisation of the bacteria.

With these first experiments we could show the binding of compound 6 by $E$. coli proteins even in very low concentrations.

In a control experiment we examined a possible unspecific binding of the biomimetic probe to the bacteria. For this experiment the acetyl protected compound 8 was used. The highest concentration of $c[8]=10^{-3} \mathrm{~mol} \mathrm{~L}^{-1}$ was used to create a clear fluorescence signal for the case that there is unspecific binding. After incubation with this derivative the bacteria were washed with buffer solution and fixed on glass slides. To our 


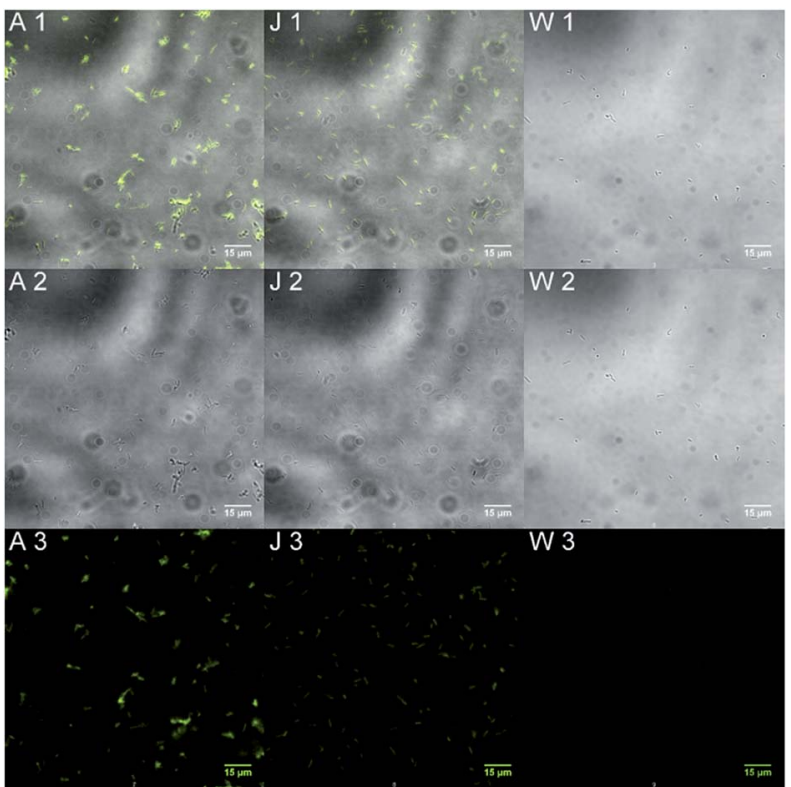

Fig. 2 Microscopy pictures of $E$. coli bacteria incubated with different concentrations of $6 . \mathrm{c}[6]=10^{-3}(\mathrm{~A}), 10^{-12}(\mathrm{~J}) \mathrm{mol} \mathrm{L}^{-1}$; without $6(\mathrm{~W})$; (1) $=2$-channel image, $(2)=$ bright field, and $(3)=$ fluorescence $\left(\lambda_{\text {ex }}=405\right.$ $\left.\mathrm{nm}, \lambda_{\mathrm{em}}=530-600 \mathrm{~nm}\right)$.

delight we could not detect any fluorescence. This means there is no unspecific binding.

To investigate the co-localisation with a better precision we used an E. coli strain DH5 $\alpha$ that was transfected with a plasmid code for pDsRed expression. Such bacteria express a red fluorescent protein (RFP) and by means of the fluorescent properties of the proteins they can be located precisely. RFP has an absorption maximum at $558 \mathrm{~nm}$ and an emission maximum at $583 \mathrm{~nm} .{ }^{15}$ The investigation was performed using the same protocol as described for the other experiments. The result of the co-localisation with compound 6 (green) and the bacteria (red) is shown in Fig. 3. Again the images were taken in bright field and fluorescence modes. The DBD derivative 6 was excited at $\lambda_{\mathrm{ex}}=405 \mathrm{~nm}$ and the protein was excited at $\lambda_{\mathrm{ex}}=543 \mathrm{~nm}$. The filter settings were chosen to get as much emission light as possible. A concentration of compound 6 of $10^{-7} \mathrm{~mol} \mathrm{~L}^{-1}$ was used to get an optimal image contrast result.

Fig. 3 shows clearly that the localization of the bacteria by either method gives the same result. Therefore it can reasonably concluded that the obtained fluorescence signals are generated from manno pyranosides 6 bound by bacteria.

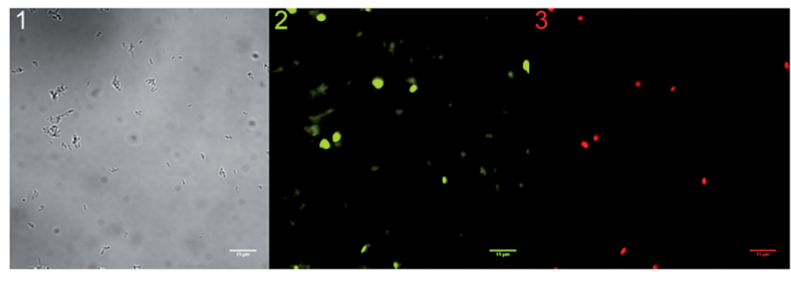

Fig. 3 Microscopy images for co-localization investigation. (1) = bright field, (2) = fluorescence $6\left(\lambda_{\text {ex }}=405 \mathrm{~nm}, \lambda_{\mathrm{em}}=530-600 \mathrm{~nm}\right)$, and $(3)=$ fluorescence $\operatorname{RFP}\left(\lambda_{\mathrm{ex}}=543 \mathrm{~nm}, \lambda_{\mathrm{em}}=560 \mathrm{~nm} \mathrm{LP}\right)$.
Another hot topic for biosensor applications is the bleaching of a fluorescent dye under real conditions. Normally, bleaching times are determined in pure solvents and those times give a good clue how a dye would behave under real conditions. However, a real biological system is much more complex so that it is suitable to investigate this system and get more exact data for the intended application. The bleaching experiments were performed with compound 6 bound on E. coli bacteria and fixed on glass slides.

For a better classification it was compared with 2 commercially available fluorescent dyes often used for microscopy. Those were Epicocconone 9, a dye that is isolated from mushrooms, and SYBR Green 10, a cyanine dye (for UV/VIS, fluorescence spectra and the formulae of $\mathbf{9}$ and $\mathbf{1 0}$ see the ESI $\dagger$ ). ${ }^{8,9,16-21}$

The E. coli strain DH5 $\alpha$ was cultivated, incubated and fixed with the comparative dyes featuring the same protocol as for compound 6 . In the following the dyes were bleached at $\lambda_{\mathrm{ex}}=$ $488 \mathrm{~nm}$ for compounds $\mathbf{9 / 1 0}$ and $\lambda_{\mathrm{ex}}=405 \mathrm{~nm}$ for compound 6 . The filters were chosen in a way that as much emission light as possible could be detected. For every investigated dye a series of 15 times was made in which images were taken in $4 \mathrm{~s}$ intervals (the decay curves of $\mathbf{6 , 9}$ and $\mathbf{1 0}$ are depicted in the ESI $\dagger$ ).

The commercially available dyes $\mathbf{9}$ and $\mathbf{1 0}$ show under real application conditions the same bleaching time dimension with $35 \mathrm{~s}$ and $50 \mathrm{~s}$. SYBR Green bleached a little faster than Epicocconone. But it is obvious that compound 6 needs much longer to bleach with a half life time of $183 \mathrm{~s}$ and thus shows a higher stability against photo-bleaching (Table 1). For the practical application higher photo-stability is an enormous advantage, because it enables a comfortable working. There is no need to do the preparation work in darkness as it was done in this experiment, nor a hurry working style is necessary when focusing the microscope.

The spectroscopic properties of compound 6 indicated that a direct staining of the bacteria should be possible without further washing procedures. Again the bacteria were incubated with different concentrations of the biomimetic probe and investigated under the microscope (Fig. 4).

At the highest concentration $\left(10^{-3} \mathrm{~mol} \mathrm{~L}^{-1}\right)$, the signal to noise ratio of bound to unbound biomimetic probe is poor as no single bacteria can be discriminated from the surrounding in the fluorescence mode. At a concentration of $c[6]=10^{-7} \mathrm{~mol}$ $\mathrm{L}^{-1}$ the signal to noise ratio is much better. The bacteria are clearly distinguishable from the buffer solution. But the best results were obtained with the lowest concentration of $c[\mathbf{6}]=$ $10^{-12} \mathrm{~mol} \mathrm{~L}^{-1}$. There is no detectable fluorescence signal from the buffer surrounding. It suggests that in low concentration a higher amount of compound $\mathbf{6}$ is bound to the bacteria. A very good signal to noise ratio of stained bacteria to buffer solution

Table 1 Photo-bleaching of compound 6 compared to 9 and 10

\begin{tabular}{lr}
\hline Compound & Bleaching half life time $[\mathrm{s}]$ \\
\hline $\mathbf{6}$ & $183.2 \pm 3.3$ \\
$\mathbf{9}$ & $50.7 \pm 1.5$ \\
$\mathbf{1 0}$ & $35.0 \pm 0.5$
\end{tabular}




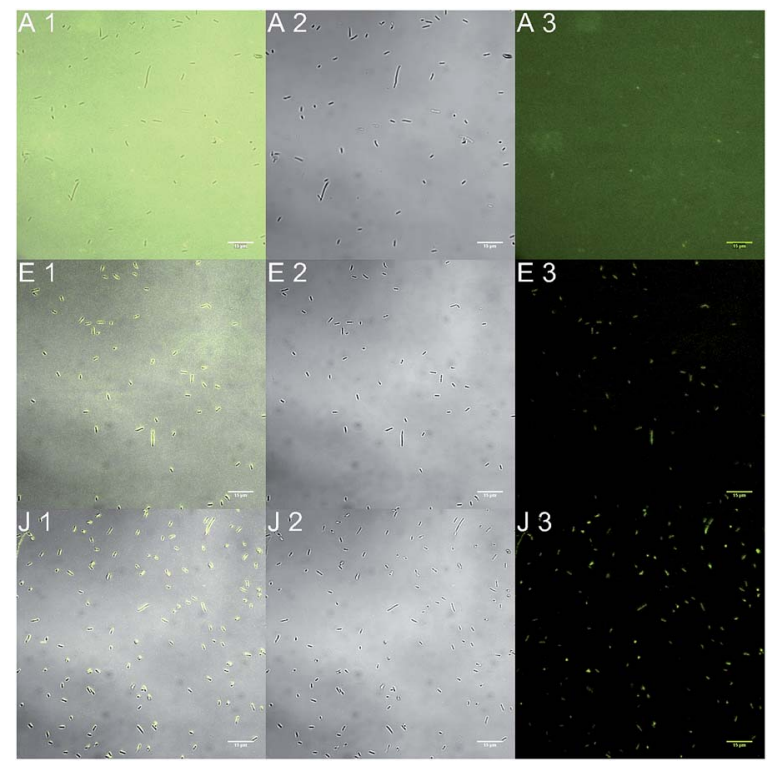

Fig. 4 Concentration series of compound 6 for direct $E$. coli staining $c[6]=10^{-3}(A), 10^{-7}(E), 10^{-12}(\mathrm{~J}) \mathrm{mol} \mathrm{L}-1(1)=2$-channel image, (2) = bright field, and $(3)=$ fluorescence $\left(\lambda_{\mathrm{ex}}=405 \mathrm{~nm}, \lambda_{\mathrm{em}}=530-600 \mathrm{~nm}\right)$.

is the result that enables a direct application of the biomimetic probe to the bacteria without any further washing procedures.

Additionally, it could be observed in this experiment that an incubation time of the bacteria with compound 6 of $\mathbf{1}$ hour is not necessary. The binding could be detected just after a few minutes, after 10 minutes the results were reproducible and even comparable with an incubation time of 1 hour. All in all it represents a very good opportunity for the so-called mix-andmeasure-process which stands for a fast on-the-spot-analytics without complex and expensive preparation and washing procedures.

In summary we could present a fast and efficient synthesis for a modular constructed biomimetic probe. This biomimetic probe has the structure of a carbohydrate fluorescent probe and we could show that it binds to $E$. coli bacteria. Due to the pronounced susceptibility of fluorescence of DBD dyes to the polarity of the microenvironment, the binding event is "switching on" the fluorescence, whereas unbound dye molecules do not fluoresce. Furthermore, the probe has a very good photo-stability and it is suitable for the direct application without further washing steps. Therefore it is very well qualified for the on-the-spot-analytics in the so-called mix-and-measureprocess. By replacing the mannose building block by more complex carbohydrates the probe could be adapted to virtually any other kind of bacteria.

\section{Notes and references}

1 (a) Topics in Fluorescence Spectroscopy. Vol. 3 Biochemical Applications, ed. J. R. Lakowicz, Kluwer, 2002; (b)
J. R. Lakowicz, Principles of Fluorescence Spectroscopy, Springer, 3rd edn, 2006; (c) J. Liu, C. Liu and W. He, Curr. Org. Chem., 2013, 17, 564.

2 P. Wessig, R. Wawrzinek, K. Möllnitz, E. Feldbusch and U. Schilde, Tetrahedron Lett., 2011, 52, 6192.

3 R. Wawrzinek, P. Wessig, K. Möllnitz, J. Nikolaus, R. Schwarzer, P. Müller and A. Herrmann, Bioorg. Med. Chem. Lett., 2012, 5367.

4 R. Wawrzinek, J. Ziomkowska, J. Heuveling, M. Mertens, A. Herrmann, E. Schneider and P. Wessig, Chem.-Eur. J., 2013, 19, 17349.

5 J. Heuveling, V. Frochaux, J. Ziomkowska, R. Wawrzinek, P. Wessig, A. Herrmann and E. Schneider, Biochim. Biophys. Acta, Biomembr., 2014, 1838, 106.

6 C. Meyners, R. Wawrzinek, A. Krämer, S. Hinz, P. Wessig and F.-J. Meyer-Almes, Anal. Bioanal. Chem., 2014, 4889.

7 R. Wawrzinek and P. Wessig, Dyes Pigm., 2015, 123, 39.

8 A. Robinson, J.-M. Fang, P.-T. Chou, K.-W. Liao, R.-M. Chu and S.-J. Lee, ChemBioChem, 2005, 6, 1899.

9 J. Coulon, I. Thouvenin, F. Aldeek, L. Balan and R. Schneider, J. Fluoresc., 2010, 20, 591.

10 S. Kuchler, M.-N. Graff, S. Gobaille, G. Vincendon, A.-C. Roche, J.-P. Delaunoy, M. Monsigny and J.-P. Zanetta, Neurochem. Int., 1994, 24, 43.

11 Y.-b. Lim, S. Park, E. Lee, J.-H. Ryu, Y.-R. Yoon, T.-H. Kim and M. Lee, Chem.-Asian J., 2007, 2, 1363.

12 D. L. Sackett and J. Wolff, Anal. Biochem., 1987, 167, 228.

13 M. Izumi, K. Fukase and S. Kusumoto, Biosci., Biotechnol., Biochem., 2001, 66, 211.

14 (a) R. Huisgen, Angew. Chem., 1963, 75, 604; (b) H. C. Kolb, M. G. Finn and K. B. Sharpless, Angew. Chem., Int. Ed., 2001, 40, 2004; (c) F. Fazio, M. C. Bryan, O. Blixt, J. C. Paulson and C.-H. Wong, J. Am. Chem. Soc., 2002, 124, 14397; (d) S. Bräse, C. Gil, K. Knepper and V. Zimmermann, Angew. Chem., 2005, 117, 5320; (e) V. D. Bock, H. Hiemstra and J. H. van Maarseveen, Eur. J. Org. Chem., 2006, 51; (f) J. Lahann, Click Chemistry for Biotechnology and Materials Science, Wiley-VCH, Weinheim, 2009.

15 G. Patterson, R. N. Day and D. Piston, J. Cell Sci., 2001, 114, 837.

16 D. R. Coghlan, J. A. Mackintosh and P. Karuso, Org. Lett., 2005, 7, 2401.

17 J. A. Mackintosh, D. A. Veal and P. Karuso, Proteomics, 2005, 5, 4673 .

18 D. S. Schabacker, I. Stefanovska, I. Gavin, C. Pedrak and D. P. Chandler, Anal. Biochem., 2006, 359, 84.

19 V. L. Singer, T. E. Lawlor and S. Yue, Mutat. Res., Genet. Toxicol. Environ. Mutagen., 1999, 439, 37.

20 I. M. Mackay, Nucleic Acids Res., 2002, 30, 1292.

21 H. Zipper, Nucleic Acids Res., 2004, 32, e103. 